\title{
Interdisciplinary Applications of Gamma-Ray Internal Conversion
}

\author{
O. Dragoun, Rez \\ (Institute of Nuclear Physics, Czechoslovak Academy of Sciences)
}

The electromagnetic interaction of an excited atomic nucleus with surrounding orbital electrons may result in the ejection of one of these electrons out of the atom. For historical reasons, the process in known as $\gamma$-ray internal conversion and the outgoing mono-energetic electron is called the internal conversion electron. By analogy with the photoelectric effect, the kinetic energy of the conversion electron is equal to the difference between the excitation energy and the binding energy in a particular atomic subshell.

Although the first mono-energetic electrons emitted in a radioactive decay were recorded by $\mathrm{O}$. von Baeyer and $\mathrm{O}$. Hahn already in 1910, it took a further 25 years to understand the nature of the phenomenon. It was proved that these electrons do not correspond to the photoelectric absorption of nuclear $\gamma$ rays; rather internal conversion and $\gamma$ ray emission are two independent and competing processes which play a dominant role in the depopulation of lowenergy excited nuclear states.

In some nuclear transitions, the conversion-electron and photon rates are comparable, in others, one of them prevails. Since there are no monopole photons, EO transitions proceed only via emission of the conversion electrons.

For an up to date account of $\gamma$-ray internal conversion theory and experiment we refer the reader to a recent critical review ${ }^{1}$ ).

\section{Internal Conversion Coefficients}

Let $\lambda_{e, i}$ and $\lambda_{\gamma}$ be the probabilities for emission of the conversion electron from the ith atomic subshell and emission of the $\gamma$ quantum, respectively, in a particular nuclear transition. The ratio

$$
\alpha_{i}=\lambda_{\mathrm{e}, i} / \lambda_{\gamma}
$$

is the subshell internal conversion coefficient (ICC). In many cases, this quantity can be calculated and measured with good accuracy which offers the possibility of gaining information on the nuclear and atomic states involved in the transition.

The theory of $\gamma$-ray internal conversion ${ }^{2}$ ) is now well developed to the first non-vanishing order of quantum theory; the contributions of higher orders are believed to be negligible in contemporary experiments. As a rule, the ICC can be calculated without any assumptions regarding the nuclear structure using only the relativistic wave functions of bound and free electrons.

The newest tables based on the Hartree-Fock-Slater atomic model, cover the ICC for all subshells of free neutral atoms of the whole periodic system and a wide range of the transition energies and multipolarities. To exploit fully the high accuracy of ICC measurements, calculations have also been carried out for particular transitions using the relativistic atomic model of Hartree and Fock. The first theoretical attempts have also been made to interpret chemical and solid-state effects in several conversion electron experiments.

\section{Nuclear Electromagnetic Transitions}

Measurements of conversion electrons started soon after the discovery of radioactivity and have since remained an important part of experimental nuclear physics. Various magnetic, semiconductor and electrostatic spectrometers were developed to measure the energies and intensities of conversion electrons emitted in both radioactive decays and nuclear reactions.

Combining conversion-electron and $\gamma$ ray spectroscopies, it was possible to determine the quantum characteristics of electromagnetic transitions in hundreds of atomic nuclei. This was an important contribution to the verification of nuclear models.

At present, transition energies are mostly measured with $\gamma$-ray spectrometers. The determination of transition multipolarities, which is needed for the determination of the spin-parity of excited nuclear states, remains the domain of conversion electron spectroscopy. This method also represents the only way to examine electric monopole transitions and transitions of very low energy. In special cases of hindered transitions, the internal conversion coefficients may be influenced by the nuclear structure, opening an additional way of investigating the nucleus.

Of particular interest is the process of inverse internal conversion when the excited atomic electrons transfer a part of their energy and momentum to the nucleus. The study of this phenomenon was motivated by the search for more effective methods of enriching ${ }^{235} \mathrm{U}$ based on the $77 \mathrm{eV}$ transition depopulating the ${ }^{235} \mathrm{U}$ isomeric state which has a half-life of $30 \mathrm{~min}$. Until now, only very weak effects have been observed in the experiments using either a laser-produced plasma or electron bombardment.

\section{Changes of Nuclear Lifetimes}

Soon after the discovery of radioactivity, careful experiments were performed on possible changes of nuclear lifetime $\tau$, and since no effect was observed, there was a general belief that nuclear half-lives cannot be influenced by any chemical or physical means. As long as half a century later it has turned out that in the case of electromagnetic transitions this need not be the case. The total probability for these transitions can be expressed as:

$$
\lambda=1 / \tau=\lambda_{\gamma}(1+\alpha)
$$

\section{Workshop on The Use of Supercomputers in Theoretical Science}

\author{
30 July - 1 August, 1984 \\ Antwerp, Belgium \\ University of Antwerp \\ "Priorij Corsendonk" Conference Centre \\ Scientific Committee : \\ J.T. Devreese (Chairman) \\ P. Van Camp \\ H. Nachtegaele \\ Further Information: \\ International Advanced Study Institute \\ c/o Mrs. R.-M. Vandekerkhof, \\ Dept. of Physics, \\ University of Antwerpen, Universiteitsplein 1, \\ B - 2610 WILRIJK (Antwerpen)
}




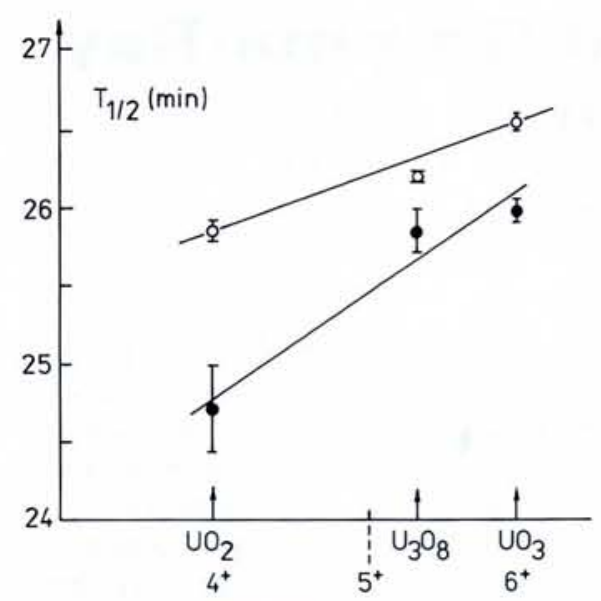

OXIDATION STATE $\longrightarrow$

Fig. 1 - Effect of the environment of uranium atoms on the $235 \mathrm{~m} U$ half-life. Radioactive sources were prepared either by wet chemistry (full circles) or by implantation of ${ }^{235 \mathrm{~m} U}$ atoms recoiled after the $\alpha$-decay of ${ }^{239} \mathrm{Pu}$ into layers of $\mathrm{UO}_{2}, \mathrm{U}_{3} \mathrm{O}_{8}$ and $\mathrm{UO}_{3}$ (empty circles). The difference in results was probably caused by perturbation of the original lattice configuration around the $235 \mathrm{~m} U$ recoils.

Here, $\alpha$ is the total ICC, i.e. the sum of the ICC over all atomic subshells for which the conversion is energetically possible. Usually, the outermost shells contribute very little to the total ICC, but even so, the electron wave functions of these shells are sensitive to the atomic environment and one can hope, at least in principle, for a measurable change of $\lambda$.

The first successful experiment was reported in 1951 by K.T. Bainbridge et al. who found a relative change of $(2.7 \pm$

Fig. 2 - The conversion electron spectrum (b) of the $77 \mathrm{eV}$ transition in ${ }^{235} \mathrm{U}$ corresponding to $235 \mathrm{~m} \mathrm{U}$ atoms deposited on the $\gamma-\mathrm{UO}_{3}$ layer. The $X$-ray photoelectron spectrum (a) of the same sample is shown for comparison.

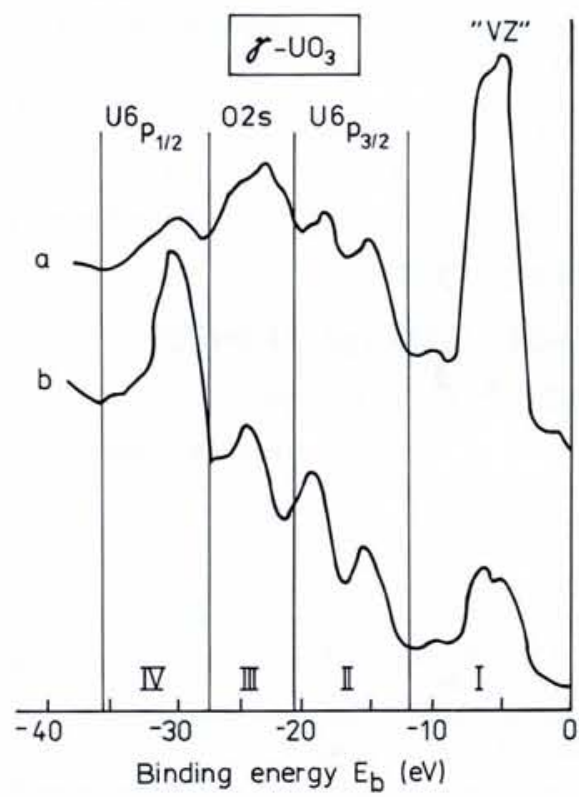

0.1) $\times 10^{-3}$ in the half-life of ${ }^{99 m} \mathrm{Tc}$ when comparing $\mathrm{KTcO}_{4}$ and $\mathrm{TC}_{2} \mathrm{~S}_{7}$ samples. The ${ }^{99} \mathrm{Tc}$ isomeric state decays via a highly converted $2.17 \mathrm{keV}$ transition. Since then, half-life changes have been observed for more than 20 isotopes. The largest variation has been found in the $77 \mathrm{eV}$ transition depopulating the isomeric level in ${ }^{235} \mathrm{U}$. Owing to the exceptionally low transition energy, the conversion of this transition proceeds only in the outermost $P$ and $Q$ shells. Halflives of $235 \mathrm{mU}$ measured for ${ }^{235 \mathrm{mUO}_{2}}$ and for $235 \mathrm{~m} \mathrm{U}$ atoms implanted into $\mathrm{Ag}$ metal differ by $9.8 \pm 1.1 \%$. As demonstrated in Fig. 1, such half-life measurements provide information on the environment of a trace amount of uranium atoms.

\section{Contact Density of Valence Shell Elec- trons}

The experimental determination of this quantity, which is not easily calculable in the framework of present models, relies on the two following facts: (1) the interaction causing the internal conversion of all electrons including the valence ones proceeds almost entirely in the vicinity of the nucleus. The ICC are proportional, with good accuracy, to the bound-electron densities at the nucleus;

(2) for low-energy transitions, the line of conversion electrons emitted from the valence shell can be distinguished in the spectra taken with high-resolution spectrometers.

In 1966, J.-P. Bocquet et al. measured the conversion-electron and Mössbauer spectra from the same samples and established the change $\Delta \rho_{5 \mathrm{~s}}(R)$ of the valence-shell density between $\mathrm{SnO}_{2}$ and white tin metal. This enabled the authors to determine the change of the nuclear charge radius $\triangle R / R$ between the excited and the ground state of ${ }^{119} \mathrm{Sn}$ which gives the calibration constant of the isomer shift.

Experiments of this type were later carried out for several other Mössbauer transitions ${ }^{3}$ ). If the $d$ - and $f$ - electrons are taking part in the chemical bond, the chemical changes of the ICC should be measured not only for the valence shell, but for the two and even three outermost shells, respectively, which is not an easy task.

\section{Unusual States of Orbital Electrons}

Grechukhin et al. demonstrated recently that the chemical environment of an atom undergoing internal conversion can influence also the number of conversion lines in the spectrum. The authors prepared a thin layer of $\gamma-\mathrm{UO}_{3}$ and deposited a small amount of the ${ }^{235 \mathrm{~m} U}$ atoms on its surface. They expected that diffusion into this layer followed by isotope exchange would aid the formation of the ${ }^{235} \mathrm{mUO}_{3}$ compound. This assumption was supported by the similarity between the Al X-ray excited photoelectron spectrum and the conversion electron spectrum of the $77 \mathrm{eV}$ transition in ${ }^{235} \mathrm{U}$ from the same sample.

In both spectra, reproduced in Fig. 2, one can see the splitting of the core $U$ $6 \mathrm{p}_{3 / 2}$ level which is caused by the electrostatic fields of ligands at the location of the uranium atoms. The line in region III of the ESCA (Electron Spectroscopy for Chemical Analysis) spectrum corresponds to photoelectrons emitted from the oxygen $2 \mathrm{~s}$ subshell of $\mathrm{UO}_{3}$. The conversion electron line in the same energy region, however, cannot be interpreted in term of the electromagnetic interaction between the ${ }^{235 \mathrm{~m} U}$ nucleus and the 2 s electrons of the surrounding $\mathrm{O}$ atom. The authors calculated that, within $1 \%$ accuracy, this interaction proceeds in a sphere of $0.5 \mathrm{pm}$ radius around the $U$ nucleus, so that the probability for the above process is several orders of magnitude smaller than that corresponding to the observed conversion line intensity. Clearly, a new orbital electron state with a binding energy of $\sim 25 \mathrm{eV}$ was identified in $\gamma-\mathrm{UO}_{3}$.

\section{Valence State of Trace Amounts of Radioactive Atoms}

The binding energies of the inner-shell electrons are known to depend slightly on the chemical environment of the atom. These energy shifts, ranging typically from 0.1 to $10 \mathrm{eV}$, are measured by means of photoelectrons emitted from a surface layer bombarded by soft X-rays. This is the basis of the modern ESCA method (for which K. Siegbahn received the Nobel Prize in 1981) applicable to almost all elements and approaching the sensitivity of $10^{-8} \mathrm{~g}$ in a $5 \mathrm{~nm}$ thick surface layer.

Environment-dependent shifts in the electron binding energies have been recognized also in a few conversion electron experiments where the authors succeeded in resolving the zero-energyloss peaks.

Recently, we have measured the $M_{4}$ and $\mathrm{M}_{5}$ conversion lines of the $2.17 \mathrm{keV}$ transition in ${ }^{99} \mathrm{Tc}$ using a cylindrical-mirror electrostatic spectrometer. We reached an overall resolution (FWHM) of 1.0 $\mathrm{eV}$ which, to our knowledge, is the best ever reported in conversion electron spectroscopy. The binding energy shifts obtained from the conversion electron spectra:

$$
\begin{aligned}
& \triangle E_{\mathrm{b}}\left(\mathrm{NH}_{4} \mathrm{TcO}_{4}-\mathrm{Tc}\right) \\
& =6.2 \pm 0.5 \mathrm{eV} \text { and }
\end{aligned}
$$




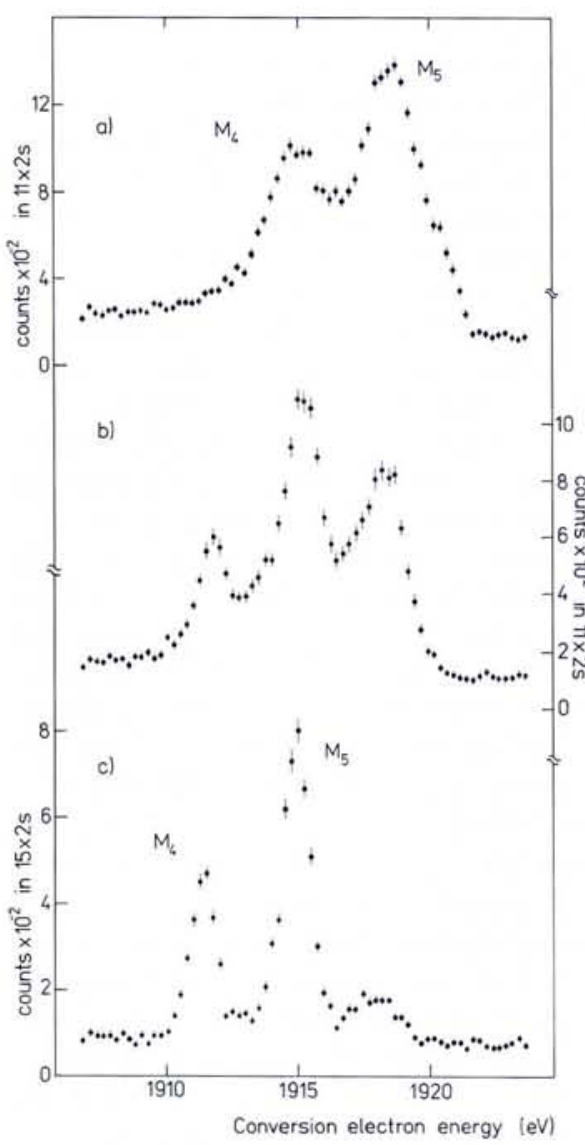

Fig. 3 - The conversion electron lines of the $2.17 \mathrm{keV}$ transition in ${ }^{99} \mathrm{Tc}$. a) Original deposit (mainly $\mathrm{TCO}_{2} \cdot 2 \mathrm{H}_{2} \mathrm{O}$ ), b) after partial oxidation (a mixture of $\mathrm{TcO}_{2} \cdot 2 \mathrm{H}_{2} \mathrm{O}$ and $\mathrm{NH}_{4} \mathrm{TcO}_{4}$ ), c) after strong oxidation (mainly $\mathrm{NH}_{4} \mathrm{TCO}_{4}$ ).

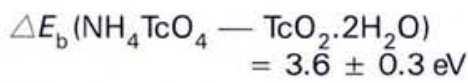

were in accord with our measurements on bulk quantities of technetium by the ESCA method. In this way we were able to determine the valence state of $10^{-11} \mathrm{~g}$ of ${ }^{99 \mathrm{~m}} \mathrm{Tc}$ deposited on a metallic foil. The strong increase of sensitivity with respect to the ESCA method is connected with a relatively high decay probability of ${ }^{99 \mathrm{~m}} \mathrm{Tc}$ which has a half-life of 6 hours only. In Fig. 3, the conversion electron lines corresponding to various chemical states of ${ }^{99 \mathrm{~m}} \mathrm{Tc}$ in the same sample are shown. Investigation of the valence state of trace amounts of ${ }^{99 \mathrm{~m}} \mathrm{Tc}$ is of interest in nuclear medicine.

\section{Mössbauer Spectroscopy of Thin Sur- face Layers}

In Mössbauer experiments involving the examination of layers of tens of $\mu \mathrm{m}$ thickness the $\gamma$-rays and X-rays transmitted through or back-scattered from the sample are measured. Many of the Mössbauer transitions are highly converted, e.g. $83 \%$ of the $14.4 \mathrm{keV}$ transitions in ${ }^{57} \mathrm{Fe}$ proceed via the emission of conversion electrons from the K-shell with a kinetic energy of $7.3 \mathrm{keV}$. Since the range of conversion electrons is much smaller than the range of $\gamma$-rays, the detection of these electrons enables one to investigate thin surface layers ( $\leq$ $0.3 \mu \mathrm{m}$ for ${ }^{57} \mathrm{Fe}$ and $\leq 3 \mu \mathrm{m}$ for ${ }^{119} \mathrm{Sn}$ ). This is the essence of conversion electron Mössbauer spectroscopy (CEMS) which is carried out in a back-scattering geometry and therefore amenable also to thick samples in a fully nondestructive way.

The conversion electrons leaving the sample surface exhibit a wide energy distribution because they are emerging from different depths of the sample and have suffered different energy losses. This fact is utilized in the depth-selective version of CEMS which supplies information about layers of definite depths and thicknesses.

As an application of the method, consider the spectra in Fig. 4 of a natural iron sample which was previously heated for $10 \mathrm{~min}$ in air at $350{ }^{\circ} \mathrm{C}$. This treatment resulted in the formation of an approximately $0.1 \mu \mathrm{m}$ thick surface oxidation layer. Accordingly, the most intensive Zeeman sextet of the Mössbauer spectrum of 1 - $2 \mathrm{keV}$ back-scattered electrons (Fig. 4b) corresponds to $\mathrm{Fe}_{2} \mathrm{O}_{3}$ while some peaks due to metallic iron and $\mathrm{Fe}_{3} \mathrm{O}_{4}$ can be seen, too. In contrast, only one sextet due to resonance absorption in much deeper layers of metallic iron is seen in the Mössbauer spectrum of back-scattered $6.3 \mathrm{keV} X$ rays (Fig. 4a). Thus CEMS is capable of revealing the presence of surface phases that would have gone undetected if the Mössbauer methods based on photon detection had been used alone.

Beta-ray spectrometers applied to energy analysis of back-scattered electrons have enabled several authors to carry out CEMS of highly enriched iron samples with a depth resolution of about $5 \mathrm{~nm}$.

In recent years, ${ }^{57} \mathrm{Fe}$ CEMS has been successfully applied to the solution of real technological problems such as:

- the formation of protective coatings on steam generator tubes in the presence of water at high temperatures and pressures;

- the characterization of steel surfaces after cold work - the austenitic could readily be distinguished from either ferritic or martensitic phases;

- the investigation of surface alloys produced by ion implantation in relation to their wear resistance.

CEMS has also been used in geochemistry where the existence of fine superparamagnetic iron particles was established in surface regions of lunar materials. In archaeology, the method has enabled investigators to characterize the iron components present in certain types of glaze on painted ceramic objects. For details we refer the reader to the review ${ }^{4}$ ) and literature cited therein.

\section{Summary and Outlook}

It will be seen that $\gamma$-ray internal conversion has followed the classic road from esoteric research to practical application. In the near future, microchannel plates serving as position-sensitive detectors in spectrometers will allow multichannel measurements of conversion electron spectra with high resolution. This will be utilized with advantage in the nuclear studies of short lived isotopes as well as in depth -selective Mössbauer spectroscopy of conversion electrons.

On the theoretical side, calculations of the internal conversion coefficients with respect to the atomic environment are necessary as well as a better understanding of the penetration of $\mathrm{keV}$ electrons through matter.

\section{REFERENCES}

1. Dragoun O., Adv. Electr. Electron Phys. 60 (1983) 1.

2. Pauli H.C., Alder K. and Steffen R.M., in The Electromagnetic Interaction in Nuclear Spectroscopy, ed. W.D. Hamilton (NorthHolland, Amsterdam) 1975, p. 341.

3. Pleiter F. and de Waard H., in Mössbauer Isomer Shifts, ed. G.K. Shenoy and F.E. Wagner (North-Holland, Amsterdam) 1978, p. 253.

4. Tricker M.J., in Mössbauer Spectroscopy and its Chemical Applications, ed. J.G. Stevens and G.K. Shenoy, Advances in Chemistry Series, 194 (Am. Chemical Soc., Washington DC) 1981, p. 63.

Fig. 4 - Mössbauer spectra of the Fe-Xrays (a) and of the low-energy electrons (b) for a slightly oxidized iron sample. Sextets of the lines due to metallic iron (I), $\mathrm{Fe}_{2} \mathrm{O}_{3}$ (II) and $\mathrm{Fe}_{3} \mathrm{O}_{4}$ (III and IV) are indicated in the lower part of the figure.

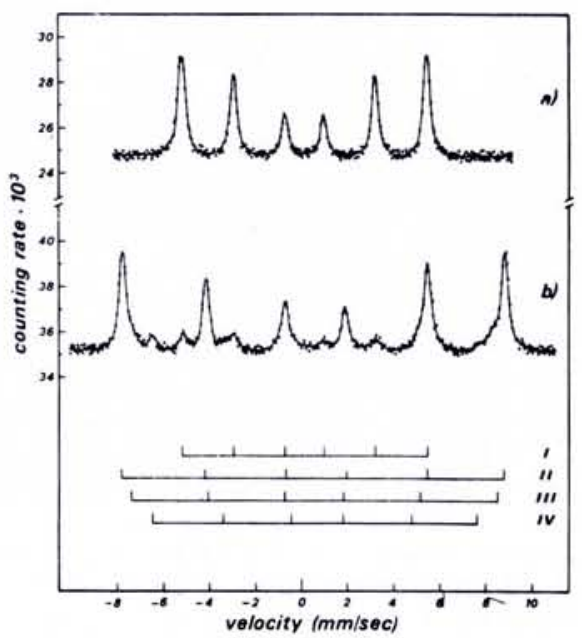

